\title{
Self-Guiding of Electromagnetic Beams in Degenerate Relativistic Electron-Positron Plasma
}

\author{
V.I. Berezhiani ${ }^{1,2}$, N.L. Shatashvili ${ }^{1,3}$ \\ ${ }^{1}$ Andronikashvili Institute of Physics, TSU, Tbilisi 017r, Georgia \\ ${ }^{2}$ School of Physics, Free University of Tbilisi, Georgia and \\ ${ }^{3}$ Department of Physics, Faculty of Exact and Natural Sciences, \\ Ivane Javakhishvili Tbilisi State University, TSU, Tbilisi 0179, Georgia
}

\begin{abstract}
The possibility of self-trapped propagation of electromagnetic beams in the fully degenerate relativistic electron-positron plasma has been studied applying Fluid-Maxwell model; it is shown that dynamics of such beams can be described by the generalized Nonlinear Schrödinger equation with specific type of saturating nonlinearity. Existence of radially symmetric localized solitary structures is demonstrated. It is found that stable solitary structures exist for the arbitrary level of degeneracy.

PACS numbers: 42.65.Jx, 52.27.Ny, 52.35.Mw, 52.38.Hb, 47.75.+f
\end{abstract}

Recent observations as well as the modern theoretical considerations indicate on the existence of superdense electron-positron plasmas in variety of astrophysical environments. The presence of e-p plasma is also argued in the $\mathrm{MeV}$ epoch of the early Universe [1]. Intense e-p pair creation takes place during the process of gravitational collapse of massive stars [2]; it is shown that in certain circumstances the gravitational collapse of the stars may lead to the charge separation with the field strength exceeding the Schwinger limit resulting in e-p pair plasma creation with estimated density to be $\sim 10^{34} \mathrm{~cm}^{-3}[3]$. The e-p plasma density can be in the range $\left(10^{30} \div 10^{37}\right) \mathrm{cm}^{-3}$ of the GRB source [4].

For highly compressed state the plasma behaves as a degenerate Fermi gas provided that averaged interparticle distance is smaller than the thermal de Broglie wavelength. As the density increases Fermi energy of the particles $\epsilon_{F}^{ \pm}=\hbar^{2}\left(3 \pi^{2} n^{ \pm}\right)^{2 / 3} / 2 m_{e}$ with $\quad+(-)$ accounting for positrons and electrons, respectively, becomes larger than the interaction energy $\left(\sim e^{2}\left(n_{0}^{ \pm}\right)^{1 / 3}\right)$ and a mutual interaction of the plasma particles becomes unimportant - plasma becomes more ideal [5]. This condition is fulfilled for a sufficiently dense fluid when $n_{0}^{ \pm} \gg\left(2 m_{e} e^{2} /\left(3 \pi^{2}\right)^{2 / 3} \hbar^{2}\right)^{3}=6.3 \cdot 10^{22} \mathrm{~cm}^{-3}$. When density increases further particle's relativistic motion shall be taken into account leading to the relativistic Fermi energy of the particles in the following form $\epsilon_{F}^{ \pm}=m_{e} c^{2}\left[\left(1+\left(R^{ \pm}\right)^{2}\right)^{1 / 2}-1\right]$, where $R^{ \pm}=p_{F}^{ \pm} / m_{e} c$, $p_{F}^{ \pm}-$is the Fermi momentum which is related to the restframe particle density by the following relation $p_{F}^{ \pm}=$ $m_{e} c\left(n^{ \pm} / n_{c}\right)^{1 / 3}$, here $n_{c}=5.9 \times 10^{29} \mathrm{~cm}^{-3}$ is the normalizing critical number-density [6]. Thus, when $n^{ \pm} \gg$ $n_{c}$ plasma turns out to be ultra-relativistic even for nonrelativistic temperature $T^{ \pm} \ll \epsilon_{F}^{ \pm}$. Here we would like to emphasize that the pair plasmas with such densities can not be in complete thermodynamic equilibrium with the photon gas into which it annihilates [7]. Equilibrium is reached within the time-period related mainly to the electron-positron annihilations. Subsequently, the thermodynamic equilibrium between pairs and photons (with zero chemical potential) will be achieved. Plasma becomes optically thick with steady state pair density defined by plasma temperature. On the other hand for high density optically thin plasma, as it was shown in [6], the e-p annihilation time becomes considerably short $\left(\tau_{a n n} \approx 0.3 \times 10^{-16} \mathrm{sec}\right.$ for the densities $\left.\sim 10^{30} \mathrm{~cm}^{-3}\right)$. However, it is still larger than the corresponding densities plasma oscillations characteristic time-scale $\left[\sim \omega_{e}^{-1}\right]$; for instance, for the densities $\left(10^{30} \div 10^{35}\right) \mathrm{cm}^{-3}$ we find that $\left(\tau_{a n n} \omega_{e}^{-1}\right)$ is in the range $(60 \div 7)[6]$ and collective plasma high frequency oscillations have enough time to manifest themselves.

Generation of high density e-p plasma is presumably augmented by production of intense pulses of $\mathrm{X}-$ and Gamma-rays. To understand the dynamics of such pulses emanating from the compact astrophysical objects as well as to study the nonlinear interactions of intense laser pulses and dense degenerate plasmas it is important to investigate the wave self-modulation and soliton formation phenomena in dense e-p plasmas. The existence of stable localized envelope solitons of electromagnetic (EM) radiation has been suggested as a potential mechanism for the production of micro-pulses in AGN and pulsars [8]. Localized solitons created in the plasma dominated era are also invoked to explain the observed inhomogeneities of the visible universe [1]. Recently, the existence of soliton-like electromagnetic distributions in a fully degenerate electron-positron plasma was shown in [6] applying relativistic hydrodynamic and Maxwell equations. For circularly polarized wave it was found that the soliton solutions exist both in relativistic as well as nonrelativistic degenerate plasmas and the possibility of plasma cavitation was also shown.

In the present paper we apply the Fluid-Maxwell model presented in [2, 6, 9] to investigate the possibility of selftrapping of intense electromagnetic pulse in transparent degenerate electron-positron (e-p) plasma in a limit of narrow pulse $L_{\perp} \ll L_{\|}$(where $L_{\|}$and $L_{\perp}$ are the characteristic longitudinal and transverse spatial dimensions of the field, respectively) to demonstrate the formation of stable 2D solitonic structures in such plasma. 
Basic set of Maxwell-Fluid equations describing the dynamics of unmagnetized fully degenerate electronpositron plasma reads as [6]:

$$
\begin{gathered}
\frac{\partial^{2} \mathbf{A}}{\partial t^{2}}-c^{2} \Delta \mathbf{A}+c \frac{\partial}{\partial t}(\nabla \varphi)-4 \pi e c\left(N^{+} \mathbf{V}^{+}-N^{-} \mathbf{V}^{-}\right)=0 \\
\Delta \varphi=-4 \pi e\left(N^{+}-N^{-}\right) \\
\frac{\partial}{\partial t}\left(G^{ \pm} \mathbf{p}^{ \pm} \pm \frac{e}{c} \mathbf{A}\right)+\nabla\left(m_{e} c^{2} G^{ \pm} \gamma \pm e \varphi\right)=0 \\
\frac{\partial}{\partial t} N^{ \pm}+\nabla\left(N^{ \pm} \mathbf{V}^{ \pm}\right)=0
\end{gathered}
$$

where $\mathbf{A}$ and $\varphi$ are the EM fields vector and scalar potentials; $\mathbf{p}^{ \pm}=\gamma^{ \pm} \mathbf{V}^{ \pm}$is the hydrodynamical momentum of particles, $\mathbf{V}^{ \pm}$is velocity, and $\gamma^{ \pm}$is a relativistic factor; $N^{ \pm}$is density in laboratory frame; the "effective mass" $G^{ \pm}$depends only on the plasma rest frame density $n^{ \pm}=N^{ \pm} / \gamma^{ \pm}$by the following simple relation $G^{ \pm}=\left[1+\left(n^{ \pm} / n_{c}\right)^{2 / 3}\right]^{1 / 2}$ which is valid for the arbitrary strength of relativity defined by the ratio $n^{ \pm} / n_{c}$.

In what follows we apply the above equations to the problem of the nonlinear self-guiding of EM beam in highly transparent e-p plasma. It is convenient to introduce the generalized momentum $\Pi^{ \pm}=G^{ \pm} \mathbf{p}^{ \pm}$and relativistic factor $\Gamma^{ \pm}=G^{ \pm} \gamma^{ \pm}$. In terms of following normalized quantities:

$$
\begin{array}{cc}
\widetilde{t}=\omega t, \quad \widetilde{\mathbf{r}}=\frac{\omega}{c} \mathbf{r}, \quad \widetilde{\mathbf{A}}=\frac{e \mathbf{A}}{m_{e} c^{2}}, \quad \widetilde{\varphi}=\frac{e \varphi}{m_{e} c^{2}}, \\
\widetilde{\boldsymbol{\Pi}}^{ \pm}=\frac{\boldsymbol{\Pi}^{ \pm}}{m_{e} c}, \quad \widetilde{n}^{ \pm}=\frac{n^{ \pm}}{n_{0}} \quad \text { and } \quad \widetilde{\mathbf{N}}^{ \pm}=\frac{\mathrm{N}^{ \pm}}{\mathrm{n}_{0}} .
\end{array}
$$

Suppressing the tilde, we arrive to the following dimensionless equations:

$$
\begin{gathered}
\frac{\partial^{2} \mathbf{A}}{\partial t^{2}}-\Delta \mathbf{A}+\frac{\partial}{\partial t}(\nabla \varphi)-\varepsilon^{2}\left(\mathbf{J}^{+}-\mathbf{J}^{-}\right)=0 \\
\Delta \varphi=\varepsilon^{2}\left(N^{-}-N^{+}\right) \\
\frac{\partial}{\partial t}\left(\mathbf{\Pi}^{ \pm} \pm \mathbf{A}\right)+\nabla\left(\Gamma^{ \pm} \pm \varphi\right)=0 \\
\frac{\partial N^{ \pm}}{\partial t}+\nabla \cdot \mathbf{J}^{ \pm}=0
\end{gathered}
$$

with $\mathbf{J}^{ \pm}=n^{ \pm} \boldsymbol{\Pi}^{ \pm} / \Gamma^{ \pm}$and $\Gamma^{ \pm}=\sqrt{\left(G^{ \pm}\right)^{2}+\left(\boldsymbol{\Pi}^{ \pm}\right)^{2}}$. Here $\epsilon=\omega_{e} / \omega \ll 1$, where $\omega$ is the frequency of EM field and $\omega_{e}=\sqrt{4 \pi e^{2} n_{0} / m_{e}}$. The above equations are similar to those derived for classical relativistic e-p plasma in [10, 11] where the "effective mass" was defined by the plasma temperature and relativistic equation of state, while in the present study $G^{ \pm}=\sqrt{1+R_{0}^{2}\left(n^{ \pm}\right)^{2 / 3}}$ [with $R_{0}=\left(\frac{n_{0}}{n_{c}}\right)^{1 / 3}$ ] which is valid for entire range of physically allowed densities. Hence, we apply below the method of multiple scale expansion of the equations in the small parameter $\epsilon$ [12]. We say that all physical variables $\left(Q=A, \varphi, \Pi^{ \pm}, \Gamma^{ \pm}, N^{ \pm}, G^{ \pm}\right)$can be expanded as

$$
Q=Q_{\{0\}}\left(\xi, x_{1}, y_{1}, z_{2}\right)+\varepsilon Q_{\{1\}}\left(\xi, x_{1}, y_{1}, z_{2}\right),
$$

where $\left(x_{1}, y_{1}, z_{2}\right)=\left(\varepsilon x, \varepsilon y, \varepsilon^{2} z\right)$ and $\xi=z-b t$ and $\left(b^{2}-1\right) \sim \varepsilon^{2}$. We further assume that EM field is circularly polarized

$$
\mathbf{A}_{\{0 \perp\}}=\frac{1}{2}(\widehat{\mathbf{x}}+i \widehat{\mathbf{y}}) A \exp (i \xi / b)
$$

with $A$ being a slowly varying envelope of EM beam.

To the lowest order in $\epsilon$, following the standard procedure, we get $\boldsymbol{\Pi}_{\{0 \perp\}}^{ \pm}= \pm \mathbf{A}_{\{0 \perp\}}$ from transverse component of Eq.(7), while the first order longitudinal component and next order Eq.(7) lead to the following: $\varphi_{(0)}=0$ and $\Gamma_{\{0\}}^{ \pm}=\Gamma_{0}=$ const ; thus the Poisson equation $\varphi_{\{0\}}=0$ gives consequently $N_{\{0\}}^{ \pm}=N_{\{0\}}$. Here $\Gamma_{\{0\}}^{ \pm}=\sqrt{G_{\{0\}}^{2}+|A|^{2}}$ with $G_{\{0\}}=\sqrt{1+R_{0}^{2} n_{\{0\}}^{2 / 3}} ;$ since fields vanish at infinity the factor $\Gamma_{0}=\sqrt{1+R_{0}^{2}} \equiv$ const. Taking into account that $n_{\{0\}}=N_{\{0\}} / \gamma=\frac{G_{\{0\}}}{\Gamma_{0}} N_{\{0\}}$ and solving algebraic relations $\Gamma_{\{0\}}^{ \pm}=\Gamma_{0}$ we obtain the following expression for the plasma density:

$$
N_{\{0\}}=\frac{1}{\delta^{3} \sqrt{1-|A|^{2} / \Gamma_{0}^{2}}}\left(\delta^{2}-|A|^{2} / \Gamma_{0}^{2}\right)^{3 / 2}
$$

where $\delta=R_{0} / \sqrt{1+R_{0}^{2}}$. Note, that Eq.(11) is valid for arbitrary level of degeneracy parameter $\delta$ provided that $|A|^{2}<R_{0}^{2}$.

For slowly varying envelope the Maxwell equation (5) reduces to $\nabla_{\perp}^{2}=\partial_{x_{1}}^{2}+\partial_{y_{1}}^{2}$ :

$$
2 i \frac{\partial A}{\partial z_{2}}+\nabla_{\perp}^{2} A+\sigma A-A \frac{2 N_{\{0\}}}{\Gamma_{0}}=0,
$$

here $\sigma=\left(b^{2}-1\right) / b^{2} \varepsilon^{2}$; if we assume that $b=\omega / k c$, than $\sigma-1 / \Gamma_{0}=0$ is nothing but the dispersion relation $\omega^{2}=k^{2} c^{2}+2 \Omega_{e}^{2}$, where $\Omega_{e}=\omega_{e} / \sqrt{\Gamma_{0}}$ is a modified plasma frequency due to degeneracy. Introducing $a=$ $A / R_{0}$, dropping subscripts for the variables $\left(z_{2}, x_{1}, y_{1}\right)$ and making self-evident re-normalization: $z=2 z / \Gamma_{0}$ and $r_{\perp}=\sqrt{2 / \Gamma_{0}} r_{\perp}$, the final equation will read as:

$$
2 i \frac{\partial a}{\partial z}+\nabla_{\perp}^{2} a+f\left(|a|^{2}\right) a=0
$$




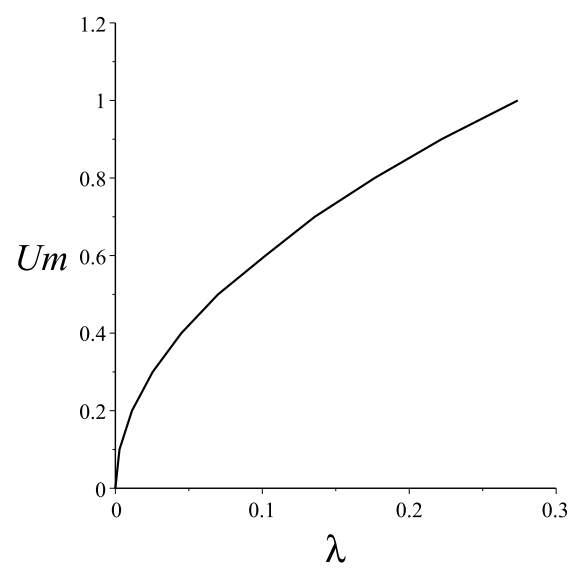

FIG. 1: The typical behavior of soliton amplitude $U_{m}$ versus propagation constant $\lambda$ for moderate degeneracy parameter case of $\delta=0.5$.

with

$$
f\left(|a|^{2}\right)=1-\frac{\left(1-|a|^{2}\right)^{3 / 2}}{\left(1-\delta^{2}|a|^{2}\right)^{1 / 2}} .
$$

Equation (13) is a Nonlinear Schrödinger Equation (NSE) with peculiar type nonlinearity function $f$. The latter is a growing function of field amplitude $|a|$ attaining its maximum value $f=1$ at $|a|=1$. For small amplitude case $(|a| \ll 1)$ the nonlinearity function reduces to $f \simeq \beta|a|^{2}$, where coefficient $\beta=0.5\left(3-\delta^{2}\right)$ varies within $(1.5-1)$ for an arbitrary level plasma degeneracy $(0<\delta<1)$. While for weak degeneracy level $(\delta \ll 1) \quad f \simeq 1-\left(1-|a|^{2}\right)^{3 / 2}$ and for relativistic degeneracy $(\delta \rightarrow 1) \quad f \simeq|a|^{2}$ at $|a| \leq 1$. NSE with various type saturating nonlinearity has been studied thoroughly in the past [1, 13 16]; our eq.(13) [with (14)] proves to be similar qualitatively though showing quantitative difference. First, one has to establish the existence and the stability of the self-trapped solutions of Equation (13). Using the axially symmetric solution ansatz in the form $a=U(r) \exp (i \lambda z)$, where $r=\left(x^{2}+y^{2}\right)^{1 / 2}$ and $\lambda$ is the so called propagation constant, the ordinary nonlinear differential equation for the radially dependent envelope $U(r)$ reduces to:

$$
\nabla_{r r}^{2} U-\lambda U+f\left(U^{2}\right) U=0
$$

with $\nabla_{r r}^{2}=\frac{d^{2}}{d r^{2}}+\frac{1}{r} \frac{d}{d r}$. We consider the lowest order nodeless (ground state) solution of above equation with maximum $U_{m}$ at the center $(r=0)$ and monotonically decreasing $(U \rightarrow 0)$ as $r \rightarrow \infty$. Maximal amplitude $U_{m}$ is determined by eigenvalue $\lambda$ which, according to [13], satisfies $0<\lambda<f_{m}$, where $f_{m}$ is a maximal value of nonlinearity function.

We have performed the numerical simulation study of the Eq.(15) with our nonlinearity (14) for arbitrary level

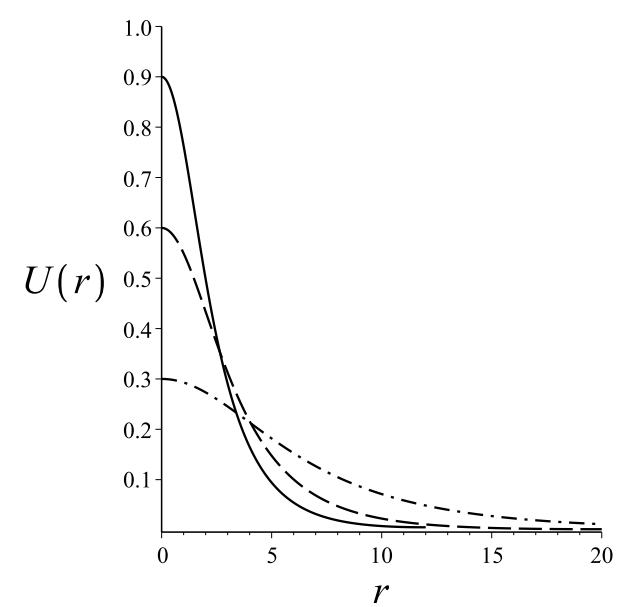

FIG. 2: Profiles of solitary structure for the variety of $U_{m}$.

of degeneracy parameter $\delta$. Despite the value of $f_{m}=1$ the allowed range of eigenvalue $\lambda$ is significantly narrow $\lambda<\lambda_{c}<1 . U_{m}$ is a growing function of $\lambda$ attaining its maximal value $U_{m}=1$ at $\lambda=\lambda_{c}$. At the same time critical value $\lambda_{c}$ decreases with $\delta$ from its maximum being 0.2912 at $\delta \ll 1$ to 0.2055 for ultrarelativistic degenerate case $(\delta \gg 1)$. The typical behavior of soliton amplitude $U_{m}$ versus propagation constant $\lambda$ is exhibited in Fig. 1 for moderate degeneracy case $\delta=0.5$ while profiles of solitary structure for the variety of $U_{m}$ is plotted in Fig.2. Important characteristics of obtained solitary solutions is a so called beam "power" defined by $P=2 \pi \int_{0}^{\infty} d r r U^{2}(r, \lambda)$. Numerical simulations show that for arbitrary level of degeneracy $\delta$ power $P$ is a growing function of $\lambda$ and, obviously, $\mathrm{U}_{\mathrm{m}}$ [such behavior for $\delta=0.5$ is presented in Fig. 3 where $P$ versus $U_{m}$ is plotted]; consequently, solitary solutions are stable against small perturbations [13]. For $\lambda \rightarrow 0\left[U_{m} \rightarrow 0\right]$ power $P \rightarrow P_{c}$ while maximal allowed value of power is achieved at $\lambda=\lambda_{c}\left[U_{m}=1\right]$; here $P_{c}$ is a critical power. Thus, self-trapped propagation solitary beam could be formed in such plasmas provided $P \geq P_{c}$.

Critical power of self-trapped beam $P_{c}$ depends on degeneracy parameter $\delta$. This relation can be obtained analytically assuming that for small amplitude case $\left(\lambda \ll 1, f \simeq \beta U^{2}\right)$ with obvious change of variables $\left[U(r)=\sqrt{\frac{\lambda}{\beta}} g(\sqrt{\lambda} r)\right]$ the equation (15) reduces to $\nabla_{r r}^{2} g-g+g^{3}=0$ [17], where $g(r)$ is a stationery Townes mode. Knowing the Power of ground state Townes mode $P_{g}=2 \pi \int_{0}^{\infty} d r r g^{2}(r)=11.69$ one can find the critical power to be $P_{c}=\beta^{-1} P_{g}=23.37 /\left(3-\delta^{2}\right)$. For the nonrelativistically degenerate case $P_{c} \sim 7.79$ while for the super-relativistic degenerate case $P_{c} \sim 11.69$.

Critical power in dimensional units can be written in a following convenient form:

$$
\left[P_{c}\right]=33 \chi \frac{\omega^{2}}{\Omega_{e}^{2}} \mathrm{GW},
$$




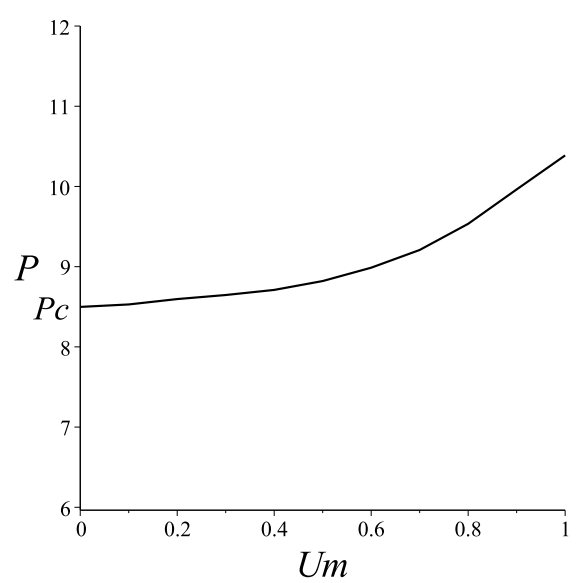

FIG. 3: $\quad$ Power $P$ versus amplitude $U_{m}$ for $\delta=0.5$ with critical power $P_{c}=8.5$.

where $\chi=R_{0}^{2} /\left(3+2 R_{0}^{2}\right)$. As we mentioned in the introduction a physically justified range of allowed plasma densities is presumably within $\left(10^{24} \div 10^{34}\right) \mathrm{cm}^{-3}\left[R_{0} \sim\right.$ $\left.1.19 \cdot 10^{-2} \div 25.69\right]$; corresponding critical power $P_{c}$ for self-trapped solution to occur is within $\left(1.6 \cdot 10^{-3} \div\right.$ 16.5) $\frac{\omega^{2}}{\Omega_{e}^{2}} \mathrm{GW}$.

At this end it is important to note that recent progress in creating dense $\mathrm{e}-\mathrm{p}$ plasmas in Laboratory conditions [18] and achievements in the development of free electron powerful X-ray sources [19] indicate that in the future the generation of the optically thin e-p plasmas can be expected with the solid state densities in the range of $\left(10^{23} \div 10^{28}\right) \mathrm{cm}^{-3}$ and above [20, 21]. For X-ray pulse with wave-length $\sim 3 \mathrm{~nm}$ interacting with $n \sim 10^{24} \mathrm{~cm}^{-3}$ density plasma the critical power becomes $\sim 194 \mathrm{MW}$. We would like to emphasize that in the case of cold nondegenerate classical plasma (temperature is zero) such effect of the existence of self-trapped solitary structures is absent.

We performed the direct simulation of derived equations to study the stability of obtained solitary solutions; analysis of this study confirms that like in the case of other type saturating nonlinearities the ground state solution is indeed stable; moreover, even for Gaussian profile initial radial distribution of field with power and amplitude close to ground state solution field quickly relaxes to the equilibrium shape structure; exception is for the initial profile with amplitude $\sim 1$ or for the initial amplitude far from ground state solution. In certain cases when initial Gaussian profile amplitude field is far from the equilibrium one the amplitude $|A|$ of the evolving field has a tendency to reach values $\geq 1$ implying that cavitation (complete expulsion of plasma from field localization area) will take place. Note, that the possibility of the existence of cavitation in classical e-p plasma has been demonstrated first time in 22]. Study of the dynamics of self-trapped solutions in cavitating regime as well as of the influence of temporal reshaping of the pulse related with group velocity dispersion is beyond the scope of present paper.

In conclusion, in present manuscript we showed that in degenerate $\mathrm{e}-\mathrm{p}$ plasma the stable self-trapped solitary 2D structures exist for arbitrary level of degeneracy. We have found the critical power for the self-guided propagation. The results of given study can be applied to understand the radiation properties of astrophysical GammaRay sources as well as may be useful to design the future laboratory experiments.

NLS would like to acknowledge the Abdus Salam International Centre for Theoretical Physics, Trieste, Italy.
[1] K.A. Holcomb and T. Tajima. Phys. Rev. D 40, 3809 (1989); V.I. Berezhiani and S.M. Mahajan. Phys. Rev. Lett. 73, 1110 (1994); Phys. Rev. E 52, 1968 (1995).

[2] N.L. Tsintsadze, P.K. Shukla and L. Stenflo. Eur. Phys. J. D. 23, 109 (2003).

[3] W.B. Han, R. Ruffini and S.S. Xue. Phys. Rev. D 86, 084004 (2012).

[4] A.G. Aksenov, R. Ruffini and G.V. Vereshchagin. Phys. Rev. E 81, 046401 (2010).

[5] L.D. Landau and E.M. Lifshitz. Statistical Physics, Pergamon Press Ltd (1980).

[6] V.I. Berezhiani, N.L. Shatashvili and N.L. Tsintsadze. Physica Scripta, 90(6), 068005 (2015).

[7] J.L. Katz. ApJ Supplement Series 127, 371 (2000).

[8] A.C.L. Chian and C.F. Kennel. Astrophys. Space Sci. 97, 9 (1983).

[9] M. McKerr, F. Haas and I. Kourakis. Phys. Rev. E 90,
$033112(2014)$

[10] L.N. Tsintsadze, Phys. Plasmas, 2, 4462 (1995).

[11] V.I. Berezhiani, S.M. Mahajan, Z. Yoshida and M. Ohhashi. Phys. Rev. E 65, 047402 (2002).

[12] G.Z. Sun, E. Ott, Y.C. Lee and P. Guzdar. Phys. Fluids 30, 526 (1987).

[13] N.G. Vakhitov and A.A. Kolokolov. Radiophys. Quantum Electron. 16, 783 (1973).

[14] V. Skarka, V.I. Berezhiani and R. Miklaszewski. Phys. Rev. E 561080 (1997);

[15] V.I. Berezhiani, S.M. Mahajan and N.L. Shatashvili. Phys. Rev. A 81(5), 053812 (2010).

[16] V.I. Berezhiani, S.M.Mahajan and N.L. Shatashvili. J. Plasma Phys. 76, 467 (2010).

[17] A.B. Borisov, A.V. Borovski, V.V. Korobkin, A.M. Prokhorov, C.K. Rhodes and O.B. Shiryaev. Sov. Phys. JETP 74(4), 604(1992) [Zh.Eksp.Teor.Fiz. 101, 1132 
(1992)].

[18] H. Chen, D.D. Meyerhofer, S.C. Wilks, R. Cauble1, F. Dollar, K. Falk, W. Goldstein, G. Gregori, A. Hazi, E. I. Moses et al., High Energy Density Physics 7, 225 (2011); G. Sarri, M.E. Dieckmann, I. Kourakis, A. Di Piazza, B. Reville, C.H. Keitel and M. Zepf. J. Plasma Physics, 81, 455810401 (2015).

[19] T. Tajima. Plasma Physics Reports, 29, 207 (2003),
[Fizika Plazmy, 29, 231 (2003)].

[20] M. Dunne. Nature Phys. 2, 2 (2006); G.A. Mourou, T. Tajima and S.V. Bulanov. 78, 309 (2006).

[21] Y. Wang, P.K. Shukla and B. Eliasson. Phys. Plasmas 20, 013103 (2013).

[22] L.N. Tsintsadze, Physica Srcipta, 50, 413, (1994). 